\title{
CT AND ULTRASOUND IN ABSCESS DETECTION AT SPECIFIC ANATOMIC SITES: A STUDY OF 198 PATIENTS
}

\author{
Robert W. Jasinski, ${ }^{*}$ Gary M. Glazer, Isaac R. Francis and Rebecca L. Harkness \\ Department of Radiology, University of Michigan at Ann Arbor. MI 48109, U.S.A.
}

(Received 17 July 1986; in revised form 26 September 1986; received for publication 18 October 1986)

\begin{abstract}
Records of 902 patients with possible abdominal abscess were reviewed and of these 198 had abscesses on at least one occasion imaged either with ultrasound (US) or computed tomography (CT). There were 235 episodes of either one or simultaneous multiple abscesses. Sensitivities of CT and US were analyzed according to anatomic site. The nature of errors made was tabulated. CT was significantly more sensitive than US for the detection of abdominal abscess. Causes of CT and US error in abscess detection are discussed, and recommendations regarding choice of exam and means of improving performance are made.
\end{abstract}

Abdominal abscess Computed tomography Ultrasound Sensitivity Enteric fistulae

\section{INTRODUCTION}

The high sensitivity of US and CT in the detection and localization of abdominal abscesses has been established [1-3]. However, there are anatomic regions and disease processes where the sensitivity of each may be less than in general. To address these issues, we retrospectively reviewed the medical, CT, and US records of 902 patients with possible abdominal abscesses.

\section{METHODS}

Patients with either clinical history or imaging results consistent with abscess were identified by review of computerized diagnostic codes and compiled CT/US reports at the University of Michigan Hospital.

Real time was performed in almost all US cases. CT was usually performed as an exam of the abdomen and pelvis.

Abscesses were classified according to anatomic compartments (Table 1). Pus identified in at least three intraperitoneal sites was classified anatomically as a diffuse abscess.

Table 1. CT and US sensitivity at anatomic sites

\begin{tabular}{lccr}
\hline & $\begin{array}{c}\text { Patients/imaging } \\
\text { Location }\end{array}$ & CT sensitivity & US sensitivity \\
\hline Intraperitoneal & & & \\
Diffuse & $28 / 28$ & $10 / 13(77 \%)$ & $11 / 25(44 \%)$ \\
Right subphrenic & $3 / 3$ & $2 / 2(100 \%)$ & $1 / 3(33 \%)$ \\
Left subphrenic & $15 / 16$ & $8 / 9(89 \%)$ & $11 / 17(65 \%)$ \\
Paracolic & $14 / 15$ & $4 / 5(80 \%)$ & $8 / 12(67 \%)$ \\
Subhepatic & $5 / 6$ & $2 / 3(67 \%)$ & $4 / 6(67 \%)$ \\
Lesser sac & $9 / 9$ & $5 / 5(100 \%)$ & $3 / 8(38 \%)$ \\
Interloop & $11 / 11$ & $3 / 5(60 \%)$ & $4 / 10(40 \%)$ \\
Pelvis & $57 / 64$ & $14 / 15(93 \%)$ & $42 / 56(75 \%)$ \\
Visceral orguns & & & \\
Hepatic & $19 / 23$ & $11 / 13(85 \%)$ & $16 / 21(76 \%)$ \\
Renal & $5 / 5$ & $0 / 0$ & $1 / 5(20 \%)$ \\
Splenic & $2 / 2$ & $1 / 1(100 \%)$ & $0 / 2(0 \%)$ \\
Pancreatic & $15 / 17$ & $11 / 12(92 \%)$ & $7 / 13(54 \%)$ \\
Retroperitoneal & $22 / 25$ & $15 / 15(100 \%)$ & $18 / 24(75 \%)$ \\
Miscellaneous & & & \\
Body Wall & $6 / 6$ & $4 / 5(80 \%)$ & $2 / 4(50 \%)$ \\
Psoas & $18 / 24$ & $21 / 23(91 \%)$ & $5 / 17(29 \%)$ \\
Incisional & $28 / 30$ & $7 / 8(88 \%)$ & $16 / 31(52 \%)$ \\
Groin & $9 / 10$ & $1 / 1(100 \%)$ & $9 / 10(90 \%)$ \\
\hline
\end{tabular}

* To whom correspondence should be addressed: Robert W. Jasinski, M.D., 2713 Kragspough Court, Holland, MI 49423, U.S.A. 
Table 2. CT vs US errors*

\begin{tabular}{|c|c|c|}
\hline \multicolumn{2}{|c|}{$\begin{array}{l}\text { US errors } \\
\text { Sensitivity- } 60 \%(158 / 264) \\
106 \text { misses in } 264 \text { imaging episodes }\end{array}$} & $\begin{array}{l}\text { CT errors } \\
\text { Sensitivity }-89 \% \text { (119 } \\
15 \text { misses in } 134 \text { imag }\end{array}$ \\
\hline $\begin{array}{l}\text { Technical } \\
\text { Interpretive } \\
\text { Perceptive } \\
\text { Communication } \\
\text { Location } \\
\text { Sampling } \\
\text { Unknown }\end{array}$ & $\begin{array}{l}62(58 \%) \\
40(38 \%) \\
6(6 \%) \\
11(10 \%) \\
7(7 \%) \\
9(8 \%) \\
2(2 \%)\end{array}$ & $\begin{array}{l}\text { Technical } \\
\text { Interpretive } \\
\text { Perceptive } \\
\text { Communication } \\
\text { Location } \\
\text { Sampling }\end{array}$ \\
\hline \multicolumn{3}{|c|}{$\begin{array}{l}\text { a Percentages indicate the frequence with which a given error typ } \\
\text { to have contributed to a misdiagnosis. In some cases, there } \\
\text { contributing factors. }\end{array}$} \\
\hline \multicolumn{3}{|c|}{ Table 3. US technical errors } \\
\hline & \multicolumn{2}{|c|}{$62 / 106$ total US errors- $(58 \%)$} \\
\hline & $\begin{array}{l}\text { Acoustic access impediment } \\
\text { Gas containing abscess } \\
\text { Obesity } \\
\text { Near field noise } \\
\text { Small size } \\
\text { Non-specificity } \\
\text { Pre-existing lesion } \\
\text { Ileus }\end{array}$ & $\begin{array}{l}43 / 62(70 \%) \\
12 / 62(20 \%) \\
9 / 62(15 \%) \\
7 / 62(11 \%) \\
2 / 62(3 \%) \\
2 / 62(3 \%) \\
1 / 62(1 \%) \\
1 / 62(1 \%)\end{array}$ \\
\hline
\end{tabular}

Errors were classified as either sampling (region of abscess not studied), technical (accepted limitation of the technique), perceptive (lesion identified in retrospect), location (anatomic location incorrect), interpretive (lesion identified, but thought to be nonabscess), communication errors (misleading use of a benign term i.e. ascites), or a combination of these.

\section{RESULTS}

Of 902 charts reviewed, 198 patients had 235 instances of one or more intra-abdominal abscess. 189 instances involved abscesses in a single location, 23 involved diffuse intraperitoneal abscesses and 23 involved abscesses in multiple locations with at least one site not peritoneal. There were 294 imaging episodes with CT and/or US exams of a given abscess.

Of 294 times that CT, US, or both were used to image an abscess, $226(77 \%)$ had surgical or autopsy proof, $48(16 \%)$ were proven by aspiration and/or culture, and $20(7 \%)$ were on the basis of clinical evaluation.

Errors were made by US in 106 of the 264 abscesses it examined (Tables 2 and 3). Technical sources of error predominated.

Errors were made in 15 of the 134 cases examined by CT (Table 2). Technical errors were primarily related to poor bowel opacification and patient movement. Interpretive errors involved abscesses mistaken for bowel or ascribed to other diseases. Perceptive errors were due to abscesses mistaken for bowel (Figs 3 and 4).

\section{Specific anatomic sites}

The use of the diffuse classification decreased the number of abscesses listed in other categories, but was necessary since both radiologists and surgeons failed to enumerate all diffuse abscess locations, precluding accurate retrospective identification of each abscess site. These diffuse abscess cases were qualitatively different than others since they were uniquely almost always related to pre-existing fluid, or intestinal leak. Of the 28 diffuse abscesses, ten were related to infection of pre-existing ascites, and fourteen were related to intestinal perforation. The most common error in this group was mislabcling infected fluid as ascites.

In the left subphrenic space, US missed six abscesses, 4 in post splenectomy patients. In the 8 asplenic patients with left subphrenic abscesses, IUS missed 4 and detected 4 .

In the pelvis, US errors were related to sampling error, echogenic abscesses, and gas containing abscesses. The general category of pelvic abscesses, especially in complex oncology cases, were not detected by US as well as CT, except those occurring in patients with noncancerous gynecologic disease. 


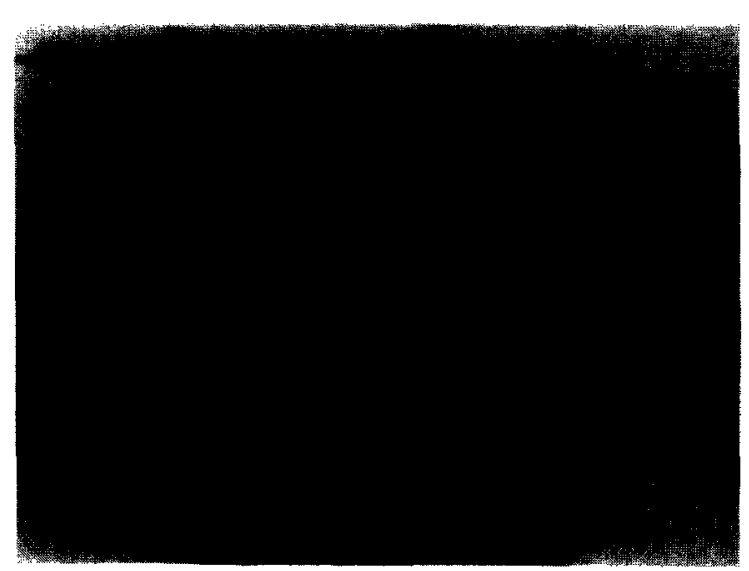

(A)

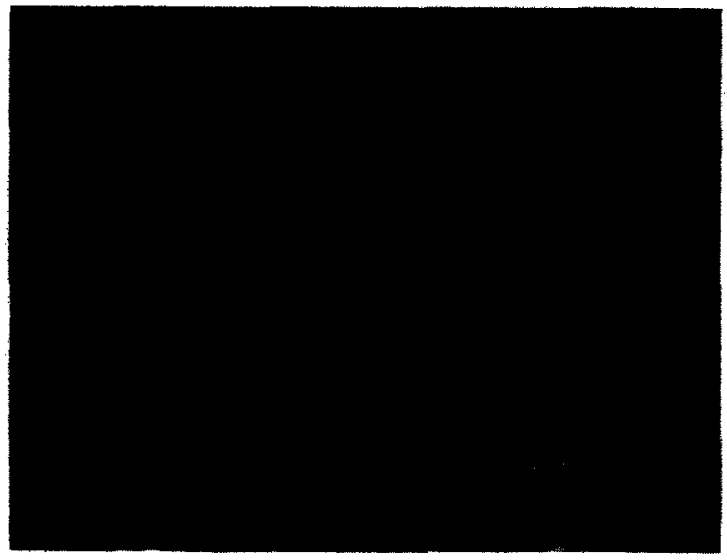

(B)

Fig. 1. (A) Left upper quadrant fluid collection thought to be stomach at real time US. (B) At CT this is a large subphrenic abscess (arrows). Medial compression of the spleen (cursor) denies its use as an acoustic window.

CT outperformed US in the retroperitoneum except for the peritransplant "perinephric" space. US was poor in diagnosing psoas abscesses, with errors related to acoustic access impediments and sampling errors occurring when the psoas was not evaluated as part of groin exams. US evaluation of localized groin abscesses due to IV drug abuse or vascular surgery was excellent. Hyperechoic abscesses and a noisy near field caused US error in incisional abscesses.

\section{CT and US both negative}

Of eight abscesses missed by both CT and US, three were communication errors labeling diffuse infected fluid as ascites. Of these, two involved infection of pre-existing ascites. Bowel perforation and peritonitis were detected by gallium scan in the third. An IN111WBC scan diagnosed a left upper quadrant abscess mistaken for bowel by CT and US. Two gas containing abscesses with enteric communications were mistaken for bowel (Fig. 4). CT and US twice failed to distinguish abscess from pre-existing tumor or pseudocyst.

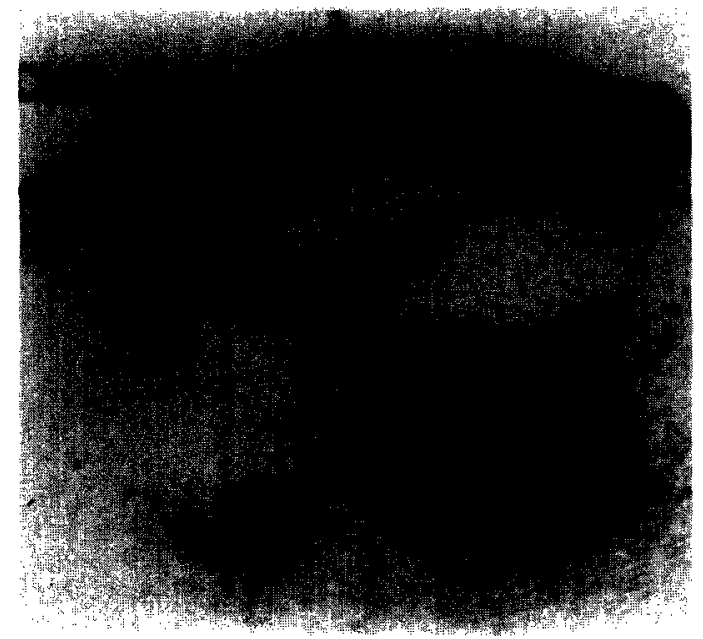

(A)

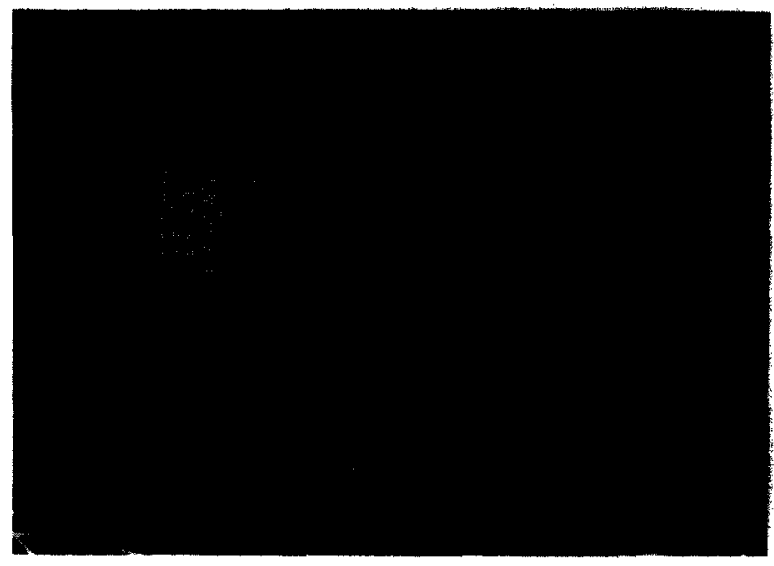

(B)

Fig. 2. (A,B) Patient with diastasis of the symphysis. The aspiration proven hematoma (black arrow) anterior to the bladder drew attention from the abscess (open arrow) compressing the rectum posterolaterally (black arrowhead). Gas and fluid caused it to mimic bowel. 


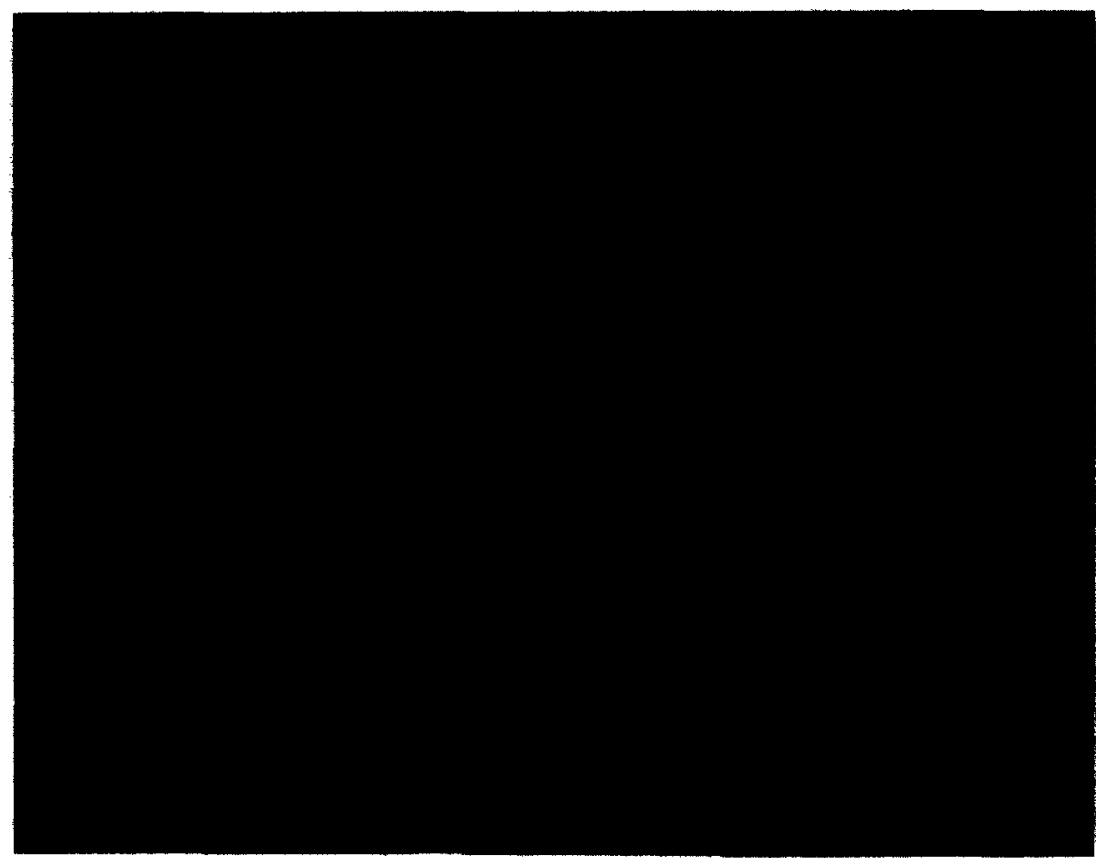

(A)

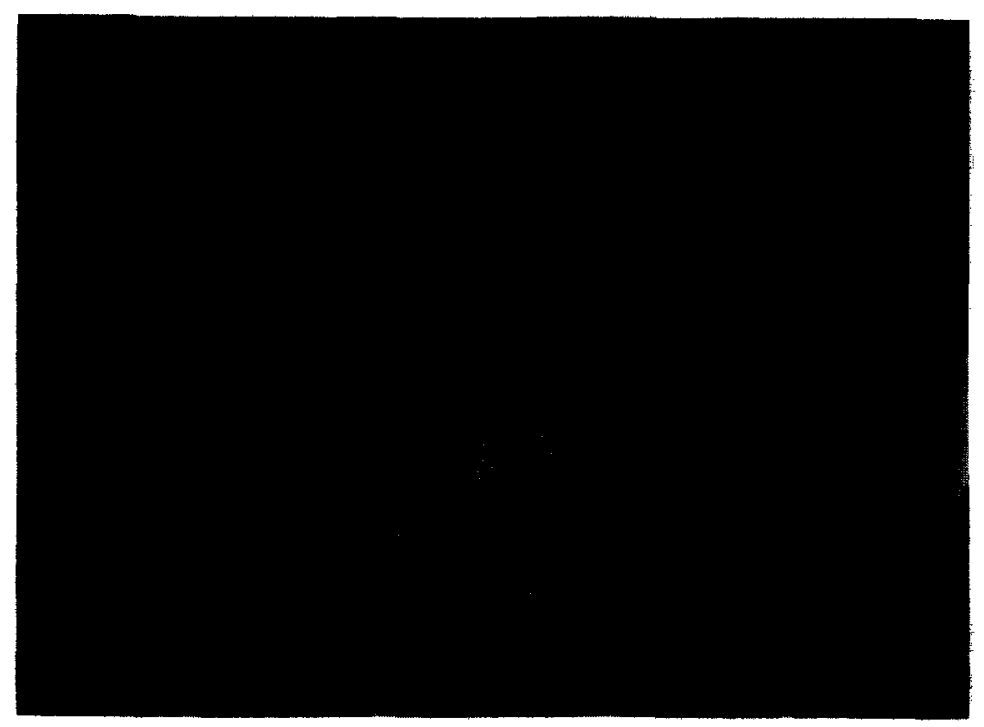

(B)

Fig. 3. Left paracolic abscess missed by CT. (A) US identified a sausage shaped fluid collection which did not peristalse (arrows), and was suspicious for abscess. (B) Inflammatory changes in the mesentery and a small abscess (small arrowhead) initially felt to be descending colon (long arrowhead) are seen in retrospect. Surgery demonstrated a paracolic abscess.

\section{DISCUSSION}

Regional differences in accuracy $[1,2,4-8]$, absolute sensitivity $[1,2,4,5,7]$, presence of wounds, tenderness, ileus [5], adjacent highly reflective and bony structures $[2,5,9]$, poor respiratory co-operation $[1,7]$, and body habitus help decide whether to use US or CT.

US directed to a specific anatomic area is more effective [5] and less expensive, but may miss unsuspected lesions due to sampling error. US sampling errors might be prevented by scanning of the pelvis and abdomen in every case $[8,10,11]$. 


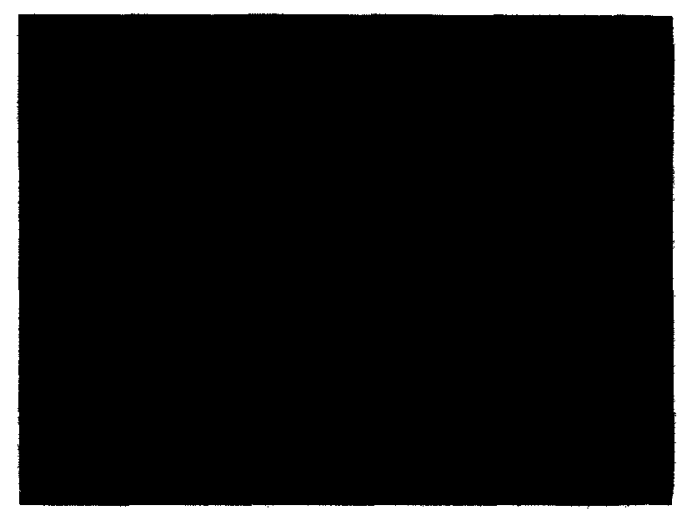

(A)

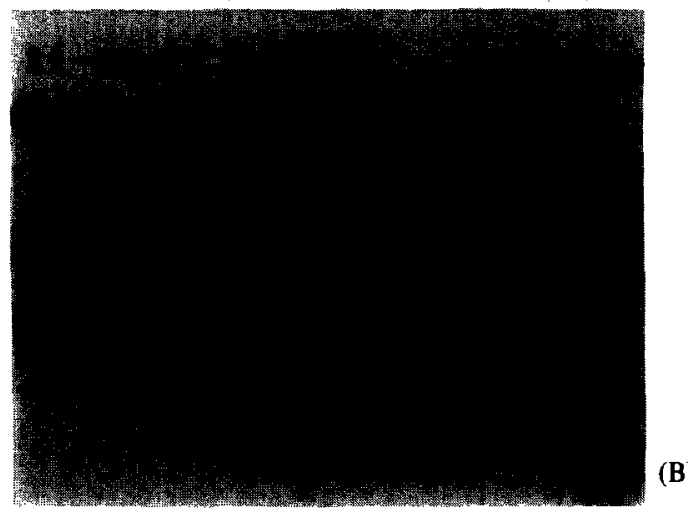

Fig. 4. Negative CT and US in a patient with Crohn's disease, a $15 \mathrm{~cm}$ abscess, and a small bowel fistula. (A) A large abscess containing predominantly air was mistaken for bowel. (B) Sagital US better demonstrates mass effect (arrowheads) superior to the bladder, thought to be an abnormal loop of small bowel.

Inherent technical limitations, primarily related to acoustic access, limit accuracy of US [2, 4, 12] and are unlikely to be overcome in a non directed US survey. CT technical errors are infrequent and usually related to patient motion or poor intestinal opacification.

US perceptive errors are uncommon. CT perceptive errors are usually due to abscesses that mimick bowel and are rare, but one of the most remediable sources of CT error. Especially in disorders associated with enteric fistulae [13], the radiologist must suspend the usual assumption that gas plus fluid or contrast equals bowel. Careful tomographic analysis with contiguous sections, review of abdominal films or digital scout view, and prone or decubitus views may prevent mislabeling an abscess as bowel [13,14]. Long air/contrast fluid levels are a clue to large abdominal abscess collections mimicking intestine. Adjacent inflammatory changes may distinguish these from bowel but are not always present [15].

CT and US interpretive errors are caused by low clinical suspicion, failure to perform needle aspiration [14], lack of tissue specificity $[3,15,13]$, and inability to distinguish new pathology from pre-existing disease $[3,9,11]$.

In some settings, such as the transplant kidney and in groin abscesses due to local factors, US sensitivity is high enough that it can be performed as the initial exam. The patient with infection of pre-existing peritoneal fluid is evaluated adequately by US with fluid aspiration. Pelvic abscess in pelvic inflammatory disease or uncomplicated post-hysterectomy cases can be reliably diagnosed by US because of few acoustic access impediments, simple clinical context, and the tendency for single abscesses confined to the pelvis.

Complex pelvic diseases, post cystectomy cases, and groin abscess without obvious local factors merit $C T$ as the initial exam.

In the pancreas, psoas, retroperitoneum [2], lesser sac, left subphrenic space, and peri-incisional regions, CT is the modality of choice because of its superior sensitivity. Inability of US to diagnose bone infection [16] makes CT preferred in the psoas or other areas where bony infection is likely. Real time transducer pressure, static scanning to display textural symmetry and psoas size, and routine 
prone scans [17] may prevent some US errors, but anteriorly placed wounds and the iliac wing impair complete US evaluation of the psoas.

CT is preferable in evaluating peri-incisional regions for abscess, especially with dehiscence. The high proportion of hyperechoic incisional abscesses makes fluid difficult to detect at US. Through transmission, an important sign of fluid [18], may not be demonstrated due to intestinal gas directly behind the abscess. The noisy near field of real time transducers poorly displays otherwise anechoic fluid. Water path scanning, or use of a high frequency real time small parts transducer with an op-site membrane [19] may help US evaluation, but lacks the larger view of static scans which allows comparison of subtle textural differences [20].

When biliary drains, biliary-enteric communication, or a fungal infection [21,22] are present or suspected, CT is preferred in evaluating the liver. Acoustic access impediments make CT preferred in the left subphrenic space, especially if the spleen is absent. Obscuring of the pancreas and lesser sac by an associated ileus, and the wide spread of inflammatory fluid make CT preferable in pancreatitis.

Patients with possible intestinal perforation should first have CT since it can detect free peritoneal air or elevated CT numbers in the fluid. At either CT or US, the finding of diffuse peritoneal fluid in a patient without pre-existing peritoneal fluid should raise suspicion of an intestinal perforation, since we and others $[23,24]$ have found that large volumes of infected peritoneal fluid may be related to enteric fistulae.

CT and US both have difficulty diagnosing abscesses in patients with enteric communications and in the background of pre-existing abnormalities such as ascites, cysts [3, 9], hydronephrosis, or neoplasms. Diagnostic aspiration may prevent such errors. Nuclear medicine has a role in the small number of patients with false negative CT and US exams, and should be strongly considered in any patient with negative CT and suspected abscess.

\section{SUMMARY}

Poor clinical clues to the abscess site and frequent occurrence of multiple abscesses favor total abdominal imaging $[1,4,12,25]$. The technical impediments to a practical US abdominal survey preclude complete confidence in a negative result. While both CT and US can reliably detect abscesses, CT is preferred for nondirected abscess search $[1,2,4,5]$, because of its high sensitivity and reliability for drainage route planning. Selection of CT and US according to patient presentation and awareness of the limitations and sources of error of each technique can minimize false negative diagnoses in cases of abdominal abscess.

Acknowledgements - Special thanks is due Dr Alex Aisen, Dr David Pennes, Dr John D. DeMott Jr, Lynn Warren, Margaret Gresham, Dianne Haupt, Cindy Fettes, Ginny Diehl, and the technical and clerical staff at the University of Michigan for their assistance. We also acknowledge the generosity of the Ex-Cello-O Corporation in providing a Laserwriter high resolution printer.

\section{REFERENCES}

1. J. T. Ferrucci and E. van Sonnenberg, Intraabdominal abscess, radiological diagnosis and treatment, J. Am. med. Assoc. 246, 2728-2733 (1981).

2. P. R. Mueller and J. F. Simeone, Intraabdominal abscesses: diagnosis by sonography and computed tomography, Radiol. Clin. N. Am. 21, 425-443 (1983).

3. J. R. Haaga, C. George, A. J. Weinstein and A. M. Cooperman, New interventional techniques in the diagnosis and management of inflammatory disease within the abdomen, Radiol. Clin. N. Am. 17, 485-513 (1979).

4. S. G. Gerzof and W. C. Johnson, Radiologic aspects of diagnosis and treatment of abdominal abscesses, Surg. Clins $N$. Am. 64, 53-65 (1984).

5. S. Saini, J. M. Kcllum, M. P. O'Leary, T. F. O’Donnell, F. P. Tally, B. Carter, R. A. Deterling and L. E. Curtis, Improved localization and survival in patients with intraabdominal abscesses, Am. J. Surg. 145, 136-142 (1983).

6. M. Crade and J. Cronan, CT and ultrasound: clinical applications in search of an abdominal abscess, Conn. Med. 44 , $418-420$ (1980).

7. M. K. Wolverson, B. Jagannadharao, M. Sundaram, P. F. Joyce, M. A. Riaz and J. B. Shields, CT as a primary diagnostic method in evaluating intraabdominal abscess, $\mathrm{Am}$. J. Roentg. 133, 1089-1095 (1979).

8. R. A. Filly, Detection of abdominal abscesses: a combined approach employing ultrasononography, computed tomography and gallium-67 scanning, J. Can. Assoc. Radiol. 30, 202-210 (1979).

9. J. R. Haaga, R. J. Alfidi, T. R. Havrilla, A. M. Cooperman, F. E. Seidelmann, N. E. Reich, A. J. Weinstein and T. F. Meaney, CT delection and aspiration of abdominal abscesses, Am. J. Roentg. 128, 465-474 (1977).

10. N. O. Whitley and C. H. Shatney, Diagnosis of abdominal abscesses in patients with major trauma: the use of computed tomography, Radiology 147, 179-183 (1983). 
11. R. L. Schapiro, L. C. Chiu and V. S. Yiu, Abdominal abscess. Diagnostic efficacy of computed tomography and comparison with ultrasonography, Computerized Tomogr. 2, 211-215 (1978).

12. R. Goldman, T. B. Hunter and K. Haber. The silent abdominal abscess: role of the radiologist, Am. J. Roentg. 141, $21-25$ (1983).

13. K. Chintapalli. M. K. Thorsen, W. D. Foley and G. F. Unger. Abdominal abscesses with enteric communications: CT findings, Am. J. Roentg. 141, 27-28 (1983).

14. R. B. Jeffrey, M. P. Federle and F. C. Laing, Computed tomography of silent abdominal abscesses, J. Comput. assist. Tomogr. 8, 67 70 (1984).

15. R. A. Dubrow and J. M. Rubin, Intraabdominal metastatic carcinoma: unusual presentation and potential pitfall in CT evaluation, J. Comput. assist. Tomogr. 6, 966-968 (1982).

16. P. R. Mueller, J. T. Ferrucci, J. Wittenberg, J. F. Simeone and R. J. Butch, Ileopsosas abscess: treatment by CT-guided percutaneous catheter drainage, Am. J. Roentg. 142, 359-362 (1984).

17. F. C. Laing and R. P. Jacobs, Value of ultrasonography in the detection of retroperitoneal inflammatory masses, Radiolog. 123, 169-172 (1977).

18. B. R. Subramanyam, E. J. Balthazar, B. N. Raghavendra, S. C. Horii, S. Hilton and D. P. Naidich, Ultrasound analysis of solid-appearing abscesses, Radiology 146, 487-491 (1983).

19. S. Fataar, H. Goodman, R. Tuft, S. Conway, T. Roman, A. Goodman, Postoperative abdominal sonography using a transsonic sealing membrane, Am. J. Roentg. 141, 565-566 (1983).

20. H. Yeh and J. G. Rabinowitz, Ultrasonography and computed tomography of inflammatory abdominal wall lesions, Radiology 144, 859-863 (1982).

21. W. Hoddick, R. B. Jeffrey, H. I. Goldberg, M. P. Federle and F. C. Laing, CT and sonography of severe renal and perirenal infections, Am. J. Roentg. 140, 517-520 (1983).

22. M. E. Berlow, B. A. Spirt and L. Weil, CT followup of hepatic and splenic fungal microabscesses, J. Comput. assist. Tomogr. 8, 4245 (1984).

23. W. A. Altemeier, W. R. Culbertson and J. P. Fidler, Giant horseshoe intra-abdominal abscess, Ann. Surg. 181, 7I6-72I (1979).

24. R. L. Nichols, Infections following gastrointestinal surgery: intra-abdominal abscess. Surg. Clins N. Am. 60, 197 212 (1980).

25. P. L. Glick, C. A. Pellegrini, S. Stein and L. A. Way, Abdominal abscess: a surgical strategy, Archs Surg. 118, 646-650 (1983).

About the Author-RoBert WILliam JASINSKi JR received the M.D. degree from the University of Chicago Pritzker School of Medicine in 1978. He completed his residency in diagnostic radioiogy at University of Chicago Hospitals and Clinics in 1982, a fellowship in CT/US at University of Michigan Hospital in 1983. and a fellowship in angiography/interventional radiology at Loma Linda University Medical Center in 1985. Much of Dr Jasinski's writing has been in the area of CT/US. Presently Dr Jasinski is enjoying private group practice in Holland, Michigan.

About the Author-GARY GLAZER is an associate professor and director of Body CT/MRI at the University of Michigan, where he has been on the faculty since 1981. He received the M.D. degree from Case Western Reserve University in 1976. At the University of California at San Francisco, he completed an internship in Internal Medicine in 1977, a residency in diagnostic radiology in 1980, and was the Clarence Heller and American Cancer Society Fellow in Body CT/Ultrasound for 1980-1981.

About the Author-ISAAC R. FranCIS received his M.B., B.S. degree from The University of Madras in 1971. After completing a residency in diagnostic radiology at Henry Ford Hospital in Detroit, Michigan, in 1981, he went on to do a fellowship in body computed tomography at The University of Michigan in Ann Arbor. where he is currently an assistant professor of radiology in the division of body CT.

About the Author-RebecCa Harkness received the A.A.S. in Nursing summa cum laude from Moraine Valley Community College in 1983, and the B.S. in Elementary Education from Valparaiso University in 1971. She has worked with a urology group and after receiving her RN, specialized in substance abuse treatment nursing. 